\title{
Cardiomiopatia/displasia arritmogênica do ventrículo direito: relato de caso
}

Arrhythmogenic right ventricular cardiomyopathy/dysplasia: case report

Miocardiopatía/displasia arritmogénica del ventrículo derecho: reporte de caso

Nalu Oliveira do Valle ${ }^{1 *}$, Marlucia do Nascimento Nobre ${ }^{1}$.

\section{RESUMO}

Objetivo: Relatar o caso clínico de um paciente com cardiomiopatia/displasia arritmogênica do ventrículo direito, do início da apresentação clínica até ser estabelecido o diagnóstico, acompanhado em um hospital de cardiologia em uma cidade do Estado do Amazonas. Detalhamento do caso: Paciente, após iniciar quadro de palpitação, dispneia e dor torácica, buscou por unidade de pronto atendimento onde foi constatada taquicardia ventricular monomórfica e iniciada cardioversão química com amiodarona, porém evoluiu para fibrilação ventricular e parada cardiorrespiratória com retorno da circulação espontânea após manobras de reanimação cardiopulmonar e desfibrilação elétrica. Posteriormente, foi submetido a exames complementares e estes somados à história clínica e exame físico permitiu diagnosticar o paciente como portador de cardiomiopatia/displasia arritmogênica do ventrículo direito; como tratamento foi submetido ao implante de cardiodesfibrilador e medicado com sotalol e losartam. Considerações finais: $O$ acompanhamento desse caso mostra uma das formas de manifestação inicial da doença, sua dificuldade diagnóstica e ainda assim a possibilidade de ser realizada sem o uso de exames invasivos.

Palavras-chave: Cardiomiopatia arritmogênica, Arritmia, Tratamento.

\begin{abstract}
Objective: To report the clinical case of a patient with arrhythmogenic right ventricular cardiomyopathy / dysplasia, from the beginning of the clinical presentation until the diagnosis was established, followed up at a cardiology hospital in a city in the state of Amazonas. Case details: Patient, after starting palpitation, dyspnea and chest pain, sought an emergency care unit where monomorphic ventricular tachycardia was found and chemical cardioversion with amiodarone was initiated, but evolved to ventricular fibrillation and cardiorespiratory arrest with return of spontaneous circulation after cardiopulmonary resuscitation maneuvers and electrical defibrillation. Subsequently, he was submitted to complementary exams and these added to the clinical history and physical exam allowed the diagnosis of the patient as having arrhythmogenic right ventricular cardiomyopathy/dysplasia; as a treatment, he underwent a cardioverter-defibrillator implant and was medicated with sotalol and losartam. Final considerations: The follow-up of this case shows one of the forms of initial manifestation of the disease, its diagnostic difficulty and yet the possibility of being performed without the use of invasive exams.
\end{abstract}

Keywords: Arrhythmogenic cardiomyopathy, Arrhythmia, Treatment.

\footnotetext{
1 Hospital Universitário Getúlio Vargas, Manaus - AM.

*E-mail: nalu.valle@yahoo.com.br
} 


\section{RESUMEN}

Objetivo: Informar el caso clínico de un paciente con miocardiopatía / displasia arritmogénica del ventrículo derecho, desde el inicio de la presentación clínica hasta que se establece el diagnóstico, seguido en un hospital de cardiología de una ciudad del Estado de Amazonas. Detalles del caso: El paciente, tras iniciar palpitaciones, disnea y dolor torácico, acudió a urgencias donde se encontró taquicardia ventricular monomórfica y se inició cardioversión química con amiodarona, pero evolucionó a fibrilación ventricular y parada cardiorrespiratoria con retorno de la circulación espontánea tras maniobras de reanimación cardiopulmonar y desfibrilación eléctrica. Posteriormente, fue sometido a exámenes complementarios y estos sumados a la historia clínica y el examen físico permitieron el diagnóstico del paciente de miocardiopatía/ displasia arritmogénica del ventrículo rerecho; como tratamiento, se sometió a un implante de desfibrilador automático y fue medicado con sotalol y losartam. Consideraciones finales: El seguimiento de este caso muestra una de las formas de manifestación inicial de la enfermedad, su dificultad diagnóstica y, sin embargo, la posibilidad de realizarse sin el uso de exámenes invasivos.

Palabras clave: Miocardiopatía arritmogènica, Arritmia, Tratamiento.

\section{INTRODUÇÃO}

A Cardiomiopatia/Displasia Arritmogênica do Ventrículo Direito (C/DVD) é uma doença com herança predominantemente autossômica dominante, embora formas recessivas associadas a um fenótipo cutâneo como a doença de Naxos e síndrome de Carvajal também sejam observadas, na qual ocorre a substituição dos cardiomiócitos por tecido fibroso e gorduroso, tal substituição pode desencadear arritmia ventricular, visto que as fibras miocárdicas sobreviventes dentro do tecido fibrogorduroso fornecem um meio para arritmias ventriculares de reentrada e dessa forma eleva o risco morte súbita (NETO EJ, et al., 2019; LI KH, et al., 2017; CORRADO D, et al., 2020).

Os desmossomos são estruturas responsáveis por manter a adesão celular e importantes para a transdução do sinal intracelular e intercelular; sendo que na fisiopatologia da C/DAVD tem-se proteínas desmossomais defeituosas ocasionando a perda da adesão entre os cardiomiócitos, seguida por inflamação, morte e produção de tecido fibrogorduroso; tal processo pode ser agravado pelo estresse na parede ventricular gerado durante atividade física, que afeta predominantemente o ventrículo direito em comparação com o ventrículo esquerdo, visto que comparativamente o primeiro é menos espesso, mais distensível e mais assimétrico (WANG W, et al., 2019; CALKINS H, 2015). Dentre as proteínas demossomais que estão mais implicadas na fisiopatologia da CDAVD temos a placoglobina, desmoplaquina, placofilina-2, desmogleína-2 e a desmocolina-2 (MARÇALO J e FALCÃO LM, 2017).

A doença possui uma prevalência de 1:2.000 a 1:5.000 e incidência de 1/1000 a 1/50 000 indivíduos, manifestando-se geralmente entre a 3-5a década (NETO EJ, et al., 2019; CALKINS H, 2015; PHILIPS B e CHENG A, 2016; MARÇALO J e FALCÃO LM, 2017). A ocorrência é rara antes dos 10 anos de idade e há uma predileção para o sexo masculino, sendo 3 homens para cada 1 mulher afetada (CABALÍN CF e STAUB KQ, 2018; MARÇALO J e FALCÃO LM, 2017). Dentre os pacientes afetados, um terço torna-se sintomático antes dos 30 anos de vida (LI KH, et al., 2017).

A C/DAVD atualmente apresenta as seguintes variantes clínicas: o fenótipo clássico da C/DAVD, ou seja, a doença que originalmente foi relatada e a mais comum, na qual ocorre o envolvimento isolado do VD; as variantes da doença biventricular, ou seja, equilibrada, dominante direita ou dominante esquerda, caracterizadas pelo acometimento paralelo predominante do Ventrículo Direito (VD) ou do Ventrículo Esquerdo (VE); e o fenótipo VE caracterizado pelo envolvimento isolado do VE, ou seja, não há envolvimento do VD clinicamente demonstrável (CORRADO D, et al., 2020).

A fim de obtermos um maior entendimento sobre a Cardiomiopatia/Displasia arritmogênica do ventrículo direito, este estudo tem como objetivo relatar o caso clínico de um paciente com tal patologia desde o início da apresentação clínica até a definição do diagnóstico. 


\section{DETALHAMENTO DO CASO}

Paciente do sexo masculino, 28 anos, em outubro de 2018, apresentou quadro de dispneia, palpitação e precordialgia, sendo que tais sintomas iniciaram após atividade física; por não haver melhora na sintomatologia buscou por atendimento em uma Unidade Hospitalar do interior do Amazonas. Na admissão, conforme resumo de transferência, apresentava ao monitor taquicardia ventricular monomórfica e por se encontrar hemodinamicamente estável foi iniciada cardioversão química com amiodarona, porém houve evolução para fibrilação ventricular e parada cardiorrespiratória; o paciente foi então submetido a manobras de reanimação cardiopulmonar e desfibrilação elétrica e desta forma ocorreu a recuperação da circulação espontânea. Permaneceu em unidade de terapia intensiva até sua transferência para um Hospital Cardiológico na região metropolitana do Estado, no qual foi admitido assintomático, porém com necessidade de investigação diagnóstica.

$\mathrm{Na}$ história patológica pregressa era sabidamente portador de hipertensão arterial sistêmica com diagnóstico realizado 6 meses antes da internação hospitalar e referiu que fazia uso irregular de hipotensor. Tabagista com carga tabágica de 12 maços/ano; na história familiar havia relato de morte súbita do avô paterno com idade inferior a 50 anos por causa cardíaca, contudo não soube informar qual a cardiopatia.

Ao exame físico: Fácies atípica; brevilíneo; bom estado geral, lúcido, orientado e colaborativo, corado; Peso: 92kg, Altura: $1.66 \mathrm{~m}$; IMC $33.4 \mathrm{~kg} / \mathrm{m}^{2}$; pressão arterial: $109 \times 74 \mathrm{mmHg}$, frequência cardíaca: $74 \mathrm{bpm}$, frequência respiratória: 18ipm; cabeça e pescoço: sem estase de jugulares; tireóide não palpável. Tórax: sem alterações; expansibilidade preservada; ausculta respiratória: murmúrio vesicular fisiológico, sem ruídos adventícios; aparelho cardiovascular: ictus cordis não visível e não palpável, ritmo cardíaco regular em dois tempos, bulhas normofonéticas, sem sopros. Abdome: semi-globoso à custa de panículo adiposo; ruídos hidroaéreos presentes, flácido, indolor à palpação e ausência de visceromegalias. Extremidades: sem edemas, pulsos amplos e simétricos.

Dentre os exames complementares ao qual o paciente foi submetido e que permitiram a elucidação diagnóstica, temos o descritivo a seguir. Na Figura 1 temos o eletrocardiograma (ECG) em $2 \mathrm{~N}$ no qual consta: ritmo sinusal, eixo:-60 -90 , FC: 60 bpm, PR:200ms, QRS: 100ms; inversão da onda T de V1-V5. Distúrbio de condução pelo ramo direito + Bloqueio divisional anterossuperior esquerdo (BDASE). Dentre as alterações encontradas neste exame temos o desvio do eixo para a esquerda, as inversões da onda $T$ (conforme indicado pelas setas) e o BDASE.

Figura 1 - Eletrocardiograma.

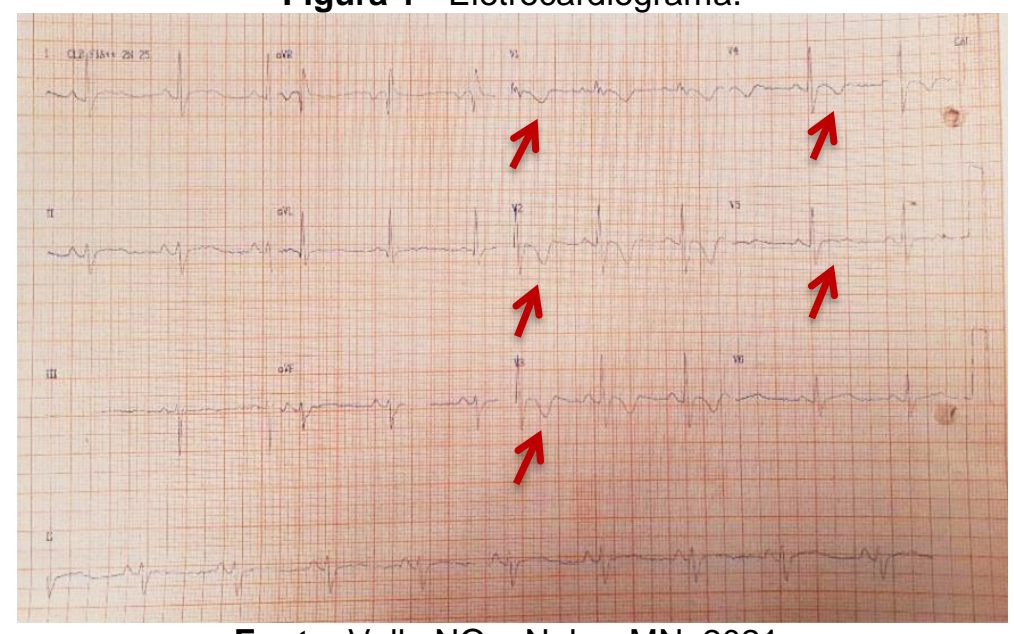

Fonte: Valle NO e Nobre MN, 2021.

Ecocardiograma Transtorácico (ECOTT): aumento do VD: 40mm, hipocinesia em porção apical do VD, Imagem da banda moderadora trabeculada, com deformidade e sobrecarga de VD. Regurgitação tricúspide de grau discreto. Fluxo em via de saída de VD e artéria pulmonar normal. Função sistólica global do ventrículo esquerdo normal. 
Holter de 24 horas: Ritmo sinusal, frequência cardíaca média: 64 bpm. Ausência de pausas maiores que 2 segundos, condução atrioventricular com comportamento normal, condução interventricular de $110 \mathrm{ms,}$ Segmentos ST sem alterações, 2 extrassístoles supraventriculares isoladas, 400 extrassístoles ventriculares isoladas, 16 episódios de extrassístoles ventriculares em salva de duas. Não houve relato de sintomas durante o período de registro.

Ressonância Nuclear Magnética Cardíaca (RNM): Disfunção ventricular sistólica direita moderada a importante (fração de ejeção do ventrículo direito: 27\%), com alterações difusas da contratilidade segmentar e diversas áreas discinéticas em parede livre do VD, durante a sístole "padrão serrilhado". Realce tardio em parede livre do VD, especialmente segmento basal. Conclusão: Paciente apresenta critérios maiores para displasia arritmogênica do VD.

Diante dos dados clínicos, eletrocardiográficos, de imagem e em conformidade com os Critérios da ForçaTarefa de 2010 para o diagnóstico da C/DAVD, o paciente foi classificado como sendo portador de doença. Durante sua internação foi submetido a implante cardiodesfibrilador (CDI), coletado material genético por swab oral e medicado com sotalol e losartana potássica.

O paciente permaneceu assintomático até sua alta hospitalar e foi orientado sobre, além de manter o tratamento medicamentoso com sotalol e losartam, a importância de não realizar atividade física extenuante ou competitiva; foi consultado se desejava ter seu caso clínico relato e mediante sua aceitação somada aos esclarecimentos realizados, formalizou seu desejo por meio do termo de consentimento livre e esclarecido (TCLE).

\section{DISCUSSÃO}

A primeira descrição clínica da C/DAVD foi realizada em 1982 em uma série de casos de pacientes que apresentavam taquiarritmia originária do ventrículo direito somada à alteração estrutural desta cavidade (GANDJBAKHCH E, et al., 2018; NETO EJ, et al., 2020). Inicialmente acreditava-se tratar-se de um defeito congênito do miocárdio do ventrículo direito sendo por tal motivo denominada de displasia, posteriormente foi classificada como sendo uma cardiomiopatia (WANG W, et al., 2019).

A apresentação clínica inicial é bastante vasta podendo ocorrer mal-estar geral, palpitações, tonturas, dor torácica, dispneia, síncope e, com menor frequência, morte súbita (MARÇALO J e FALCÃO LM, 2017). Sendo que as manifestações mais frequentes da doença são palpitações (30-60\%), tontura (20\%) e síncope (10$30 \%$ ), esses sintomas estão associados com arritmias ventriculares não sustentadas ou sustentadas, sendo que até 19\% dos pacientes com C/DVD apresentam parada cardíaca (CORRADO D, et al., 2017; GUPTA R, et al., 2017). Foi possível constatar que o quadro clínico apresentado pelo paciente preenche critérios com sinais e sintomas mais clássicos descritos na literatura para esta doença.

Dentre as alterações eletrocardiográficas que podem ser encontradas a presença de onda $T$ invertida de V1 até V3 na ausência de bloqueio completo do ramo direito é um dos principais critérios, sendo o mais comum ao ECG (WANG W, et al., 2019). No caso relatado, a inversão da onda T estendia-se até V5 o que é um indicativo de anormalidade estrutural grave e envolvimento do lado esquerdo (WANG W, et al., 2019). O bloqueio completo ou incompleto do ramo direito, estando o último presente no eletrocardiograma do paciente, é um achado comum, em especial nos pacientes com grave comprometimento estrutural (CALKINS H, 2015). A onda Epsilon, uma deflecção de baixa frequência que ocorre ao término do QRS e antes da onda $T$, decorre da pós-excitação ventricular e está presente em $30 \%$ dos portadores da C/DAVD sendo mais bem vista de V1-V3 (LI KHC, et al., 2018). A onda Epsilon não se fazia presente no eletrocardiograma do paciente deste caso clínico.

O laudo do ecocardiograma transtorácico foi sugestivo de C/DAVD considerando a anormalidade global e segmentar da parede ventricular direita em associação à dilatação da mesma. Hipertrofia de VD, disfunção sistólica e dilatação da via de saída do VD são também alterações que podem ser encontradas no ECOTT de pacientes com essa doença (CALKINS H, 2015). Para segmento ecocardiográfico destes pacientes e na dependência da idade, da genética e estado clínico, recomenda-se que seja realizado com intervalo de 1-3 anos (CORRADO D, et al., 2020). 
A Ressonância Magnética Cardíaca constitui-se o exame de imagem padrão ouro para o diagnóstico de C/DAVD, por permitir avaliar a presença de acinesia, discinesia, hipocinesia, micro ou macroaneurismas, fração de ejeção e dilatação do VD. O gadolínio possibilita observar a substituição fibrogordurosa no miocárdio e correlaciona-se com a biópsia endomiocárdica, identificando as áreas de fibrose e dessa forma fazendo mapeamento das regiões arritmogênicas (MAST TP, et al., 2015).

No caso relatado, o paciente apresentava redução da fração de ejeção do VD, discinesia e o chamado "padrão serrilhado", devido a um enrugamento na parede do VD conhecido como "sinal do acordeon" (NETO EJ, et al., 2019). Por ser um exame de custo elevado e com execução demorada deverá ser repetido se houver piora clínica significativa, alteração no ECG ou no ECOTT ou arritmias (CORRADO D, et al., 2020).

O paciente não foi submetido à biopsia cardíaca em decorrência da mesma ser raramente realizada em decorrência da sua baixa sensibilidade e isso se deve não só pelo fato das lesões da C/DAVD serem segmentares, mas também porque as amostras são geralmente obtidas a partir da região do septo, por razões de segurança, e não na parede livre do ventrículo direito, local de maior acometimento pela doença (NETO EJ, et al., 2019).

O diagnóstico da C/DAVD baseia-se na padronização estabelecida pelos Critérios da Força-Tarefa de 2010, o qual consta de um sistema de pontuação que leva em consideração: alteração estrutural do VD, caracterização tecidual, anormalidades de repolarização, anormalidades de despolarização, arritmias ventriculares e história familiar. Cada categoria possui critérios maiores, com valor de 2 pontos e menores, com valor de 1 ponto. Será considerado como portador de C/DAVD o paciente que apresenta um total de 4 pontos (WANG W, et al., 2019).

Neste relato de caso o paciente apresentou dois critérios maiores os quais foram: discinesia regional do VD com fração de ejeção do VD $\leq 40 \%$, presente no exame de ressonância magnética cardíaca, e ondas $T$ invertidas nas precordiais direitas (V1, V2 e V3) constatado no eletrocardiograma; dessa forma totalizando 4 pontos o que o enquadra, conforme TF 2010, como sendo portador da C/DAVD.

O tratamento não medicamentoso constituiu-se de orientação sobre a contraindicação para atividades extenuantes, por agravarem a progressão da doença em modelos humanos e animais, ou participação em esportes competitivos, visto que tal prática dobra o risco de taquiarritmias ventriculares e morte súbita, quando comparado aos esportes recreativos (PARK KH, et al., 2017; RUWALD AC, et al., 2015).

Como opções farmacológicas poderão ser utilizados os antiarrítmicos como sotalol, cloridrato de amiodarona e betabloqueadores (NETO EJ, et al.,2019). Os bloqueadores do canal de cálcio podem ser eficazes em pacientes selecionados (ERMAKOV S e SCHEINMAN M, 2015). Flecainida em combinação com sotalol / metoprolol pode ser uma estratégia adequada para o controle de arritmias ventriculares em pacientes refratários à terapia com agente único e / ou ablação por cateter (ERMAKOV S, et al., 2017).

Nos casos de taquicardia ventricular sustentada, síncope de origem arrítmica, alto grau de ectopia ventricular, taquicardia ventricular não sustentada ou morte súbita abortada há indicação de implante do CDI (CALKINS H, 2015; CORRADO D, et al., 2017).

Para o paciente apresentado neste caso clínico foi optado por tratamento medicamentoso com sotalol, por apresentar menos efeitos colaterais quando comparado com a amiodarona (PHILIPS B e CHENG A, 2016). O implante de cardiodesfibrilador foi indicado considerando as arritmias ventriculares graves e a morte súbita abortada.

O acompanhamento desde caso, além de ampliar significativamente o conhecimento sobre esta patologia e entendimento sobre uma de suas formas de apresentação inicial, demonstra quão desafiador é estabelecer o diagnóstico da cardiomiopatia/displasia arritmogênica do ventrículo direito, visto trata-se de uma doença rara e à qual não possui um único exame definidor do diagnóstico, mas que através de uma adequada anamnese e exame físico somado a exames não invasivos foi possível estabelecer o diagnóstico de uma doença grave e com risco de morte súbita. 


\section{REFERÊNCIAS}

1. CABALÍN CF, STAUB KQ. Miocardiopatía arritmogenica del ventrículo derecho: particularidades del enfrentamiento diagnóstico en pediatría. Rev Urug Cardiol., 2018; 33(3): 360-373.

2. CALKINS H. Arrhythmogenic right ventricular dysplasia/cardiomyopathy: three decades of progress. Circ J., 2015; 79(5): 901-913.

3. CORRADO D, et al. Arrhythmogenic cardiomyopathy. Circ Res., 2017; 121(7): 784-802.

4. CORRADO D, et al. Arrhythmogenic Right Ventricular Cardiomyopathy. N Engl J Med., 2017; 376(15): 1489-1490.

5. CORRADO D, et al. Arrhythmogenic right ventricular cardiomyopathy: evaluation of the current diagnostic criteria and differential diagnosis Domenico European Heart Journal, 2020; 41(14): 1414-1429.

6. GANDJBAKHCH E, et al. Clinical Diagnosis, Imaging, and Genetics of Arrhythmogenic Right Ventricular Cardiomyopathy/Dysplasia. Journal of the American College of Caediology, 2018; 72(7): 784-804

7. ERMAKOV S, et al. Use of flecainide in combination antiarrhythmic therapy in patients with arrhythmogenic right ventricular cardiomyopathy. Heart Rhythm., 2017;14(4):564-569.

8. ERMAKOV S, SCHEINMAN M. Arrhythmogenic right ventricular cardiomyopathy- antiarrhythmic therapy. Arrhythm Electrophysiol Rev., 2015; 4(2): 86-89.

9. GUPTA R, et al. Comparison of Features of Fatal Versus Nonfatal Cardiac Arrest in Patients With Arrhythmogenic Right Ventricular Dysplasia/Cardiomyopathy. Am J Cardiol., 2017; 120(1): 111-117.

10. LI KHC, et al. Arrhythmogenic right ventricular cardiomyopathy/dysplasia (ARVC/D) in clinical practice. Journal of Arrhythmia, 2018; 34(1): 11-22.

11. MARÇALO J, FALCÃO L.M. Miocardiopatia arritmogénica do ventrículo direito - particularidades de um caso. Rev Port Cardiol., 2017; 36(3): 1-10.

12. MAST TP, et al. Left Ventricular Involvement in Arrhythmogenic Right Ventricular Dysplasia/Cardiomyopathy Assessed by Echocardiography Predicts Adverse Clinical Outcome. J Am Soc Echocardiogr., 2015; 28(9): 1103-1113.

13. NETO EJ, et al. Cardiomiopatia/Displasia Arritmogênica do Ventrículo Direito (C/DAVD) - O Que Aprendemos após 40 Anos do Diagnóstico desta Entidade Clínica. Arq Bras Cardiol., 2019; 112(1): 91-103.

14. PARK KH, et al. Effectiveness of implantable cardioverter-defibrillator therapy for heart failure patients according to ischemic or non-ischemic etiology in Korea.Korean. Circ J., 2017; 47(1): 72-81.

15. PHILIPS B, CHENG A. 2015 update on the diagnosis and management of arrhythmogenic right ventricular cardiomyopathy. Curr Opin Cardiol., 2016; 31(1): 46-56.

16. RUWALD AC, et al. Association of competitive and recreational sport participation with cardiac events in patients with arrhythmogenic right ventricular cardiomyopathy: results from the North American multidisciplinary study of arrhythmogenic right ventricular cardiomyopathy. Eur Heart J., 2015; 36(27): 1735- 1743.

17. WANG W, et al. Diagnostic and therapeutic strategies for arrhythmogenic right ventricular dysplasia/cardiomyopathy patient. Europace, 2019; 21(1): 9-21. 